\title{
Protocolo de Mapeamento Sistemático para Busca de Aplicativos de Saúde em Repositórios Não-acadêmicos*
}

\author{
Luis F. de Lima ${ }^{1}$ e Leticia M. Peres ${ }^{1}$ \\ ${ }^{1}$ Programa de Pós-Graduação em Informática (PPGInf) \\ Universidade Federal do Paraná (UFPR) \\ Curitiba - PR - Brasil \\ \{lflima, lmperes\}@inf.ufpr.br
}

\begin{abstract}
Resumo. Este artigo apresenta um trabalho em andamento de adaptação de um protocolo de mapeamento sistemático da literatura para o levantamento de aplicativos de software de saúde em repositórios não-acadêmicos de aplicativos e código-fonte. Foram elaborados dois guias de utilização do protocolo ajustados para profissionais de saúde e desenvolvedores de software. Espera-se que estes guias auxiliem na obtenção de resultados mais rigorosos e imparciais nestas pesquisas. Um estudo de viabilidade será conduzido na próxima etapa deste trabalho para o refinamento do protocolo e dos guias de aplicação.
\end{abstract}

\section{Introdução}

Software de código aberto é um software disponibilizado sob licenças que permitem liberdade de uso, cópia, modificação e distribuição por qualquer indíviduo e para qualquer finalidade [Laurent 2004]. Na saúde, a implantação deste tipo de software aumenta a visibilidade de políticas públicas de saúde, ações e utilização de recursos que buscam ampliar a participação e controle social e democratizar o acesso à informação [Brasil 2008]. Ainda, contribui para a modernização, integração e manutenção de sistemas de saúde com menores custos [Reynolds and Wyatt 2011]. Os benefícios do software de código aberto são viabilizados pela distribuição de código-fonte de programas, bem como seu formato executável, para uso em dispositivos móvel ou desktop [Hexsel 2002]. Essa distribuição ocorre geralmente em repositórios de aplicativos, em versões gratuitas ou pagas, ou em repositórios abertos de código-fonte. Estes repositórios não-acadêmicos são considerados como fonte de literatura cinzenta ${ }^{1}$ (LC) em mapeamentos sistemáticos da literatura (MSL) e a identificação de aplicações e ferramentas de software ${ }^{2}$ hospedadas nestes repositórios pode ser dificultada neste tipo de estudo [Pappas and Williams 2011].

A LC é frequentemente utilizada para compartilhar ideias e experiências da indústria que comumente não são publicadas em literatura acadêmica revisada por pares [Garousi et al. 2020]. Assim, pode ser considerada uma fonte valiosa de conhecimento, fornecendo evidências práticas, atualizadas e de fácil acesso e contribuindo para complementar resultados obtidos em estudos experimentais [Kamei et al. 2020]. Trabalhos de MSL englobando LC vêm ocorrendo [Yasin et al. 2020, Kamei et al. 2021],

\footnotetext{
*Os artefatos de revisão aberta por pares deste artigo estão disponíveis em https : / / zenodo.org/ communities/opensciense2021

${ }^{1}$ Literatura cinzenta (do inglês, grey literature) é definida como "o que é produzido em todos os níveis do governo, institutos, academias, empresas e indústria, em formato impresso e eletrônico, mas que não é controlado por editores científicos ou comerciais" [Program 1999].

${ }^{2}$ Neste trabalho, o termo 'aplicativo' será utilizado para se referir a aplicações e ferramentas de software.
} 
porém sem considerar os repositórios de aplicativos e código-fonte em seus estudos. Buscas de aplicativos de saúde nestes repositórios são encontradas na literatura [Brown et al. 2020, Marcelo et al. 2020, Morais et al. 2020, Trecca et al. 2021], porém sem o apoio e a adaptação completa de um protocolo de MSL previamente definido e adaptado para o ambiente de repositórios não-acadêmicos.

Mediante este contexto, o presente trabalho apresenta adaptações de um MSL visando obter um protocolo para buscas de aplicativos de saúde em repositórios nãoacadêmicos. Os ajustes realizados objetivam o uso do protocolo por profissionais de saúde e desenvolvedores de software, para pesquisas em repositórios de aplicativos e códigofonte. Este artigo está estruturado da seguinte forma: A Seção 2 apresenta o referencial teórico. A Seção 3 apresenta as adaptações do protocolo de MSL. A Seção 4 apresenta trabalhos relacionados. Por fim, as considerações finais são apresentadas na Seção 5.

\section{Referencial teórico}

O MSL é um estudo que auxilia a investigação de pesquisas relevantes sobre um tópico contribuindo na estruturação de uma área de pesquisa [Kitchenham and Charters 2007]. Os MSLs são realizados com metodologias formalizadas em um protocolo contendo procedimentos com aspectos de confiabilidade, rigor, auditoria e imparcialidade para a identificação, agrupamento e análise de estudos. Ainda, indicam os passos para a divulgação das decisões da pesquisa e os resultados obtidos, possibilitando a reexecução e reprodução do estudo. Os MSLs buscam a identificação de trabalhos oriundos de revistas e eventos, como congressos e simpósios, revisados por pares e indexados em bases acadêmicas. Assim, a LC é geralmente excluída por estes estudos. Um MSL é estruturado nas três etapas descritas a seguir [Kitchenham and Charters 2007].

A etapa 1) Planejamento se inicia com a 1.a) definição do objetivo do MSL. Paradigmas como o "Goal-Question-Metric" (GQM) [Basili and Rombach 1988] podem auxiliar essa definição. Em seguida ocorre a 1.b) definição das questões de pesquisa (QPs) que devem determinar o que será respondido ao final do MSL. São elaboradas uma QP principal e subquestões. Esta etapa é finalizada com a 1.c) definição da estratégia de buscas, que envolve a escolha das bases de dados para as buscas e a definição das palavras-chave que originam as expressões de busca. Metodologias como a "Population, Intervention, Comparation, Outcome, Context" (PICOC) [Kitchenham and Charters 2007] podem ser usadas nesta definição. A etapa 2) Condução se inicia com a 2.a) definição da estratégia de seleção, que envolve a elaboração dos critérios de inclusão (CIs) e critérios de exclusão (CEs). Após essas definições, são realizadas as buscas nas bases de dados que resultam na amostra inicial de artigos. O processo de seleção de artigos ocorre em duas fases: na fase de seleção preliminar ( $1^{\circ}$ filtro) são selecionados os artigos que se enquadram no escopo da pesquisa com a aplicação dos critérios definidos e a leitura de título, resumo e palavras-chave de cada artigo da amostra inicial; na etapa de seleção final ( $2^{\circ}$ filtro) ocorre a leitura completa dos artigos selecionados no $1^{\circ}$ filtro utilizando os mesmos critérios de seleção resultando na amostra final preliminar de artigos. Esta amostra pode ser complementada com o processo de snowballing [Wohlin 2014], que se trata de uma busca opcional nas referências e/ou citações dos artigos selecionados. Opcionalmente, pode-se realizar a 2.b) avaliação de qualidade com a exclusão de artigos que não atendam aos itens definidos para verificação da qualidade. Com a amostra final definida, ocorre a 2.c) extração de dados das informações 
gerais do artigo e das QPs com o auxílio de um formulário de extração e a 2.d) análise de dados com a apresentação de resultados em tabelas, gráficos e considerações sobre as QPs. A etapa 3) Publicação envolve a escrita e a divulgação do protocolo e dos resultados do MSL e deve fornecer condições para sua repetibilidade e reprodutibilidade.

\section{Adaptação do protocolo de MSL}

As adaptações foram realizadas a partir do protocolo de MSL de [Petersen et al. 2015] e de abordagens de levantamento de aplicativos de saúde em repositórios apresentadas na Seção 4. A Figura 1 apresenta uma visão geral do protocolo e destaca as atividades com adaptações. Foram realizados ajustes nas atividades 1.c, 2.a, 2.b e 2.c. As demais atividades e a etapa 3) Publicação não sofreram alterações e devem ser conduzidas conforme a descrição apresentada na Seção 2. Os ajustes no protocolo descritos a seguir foram definidos para profissionais da saúde e desenvolvedores de software.

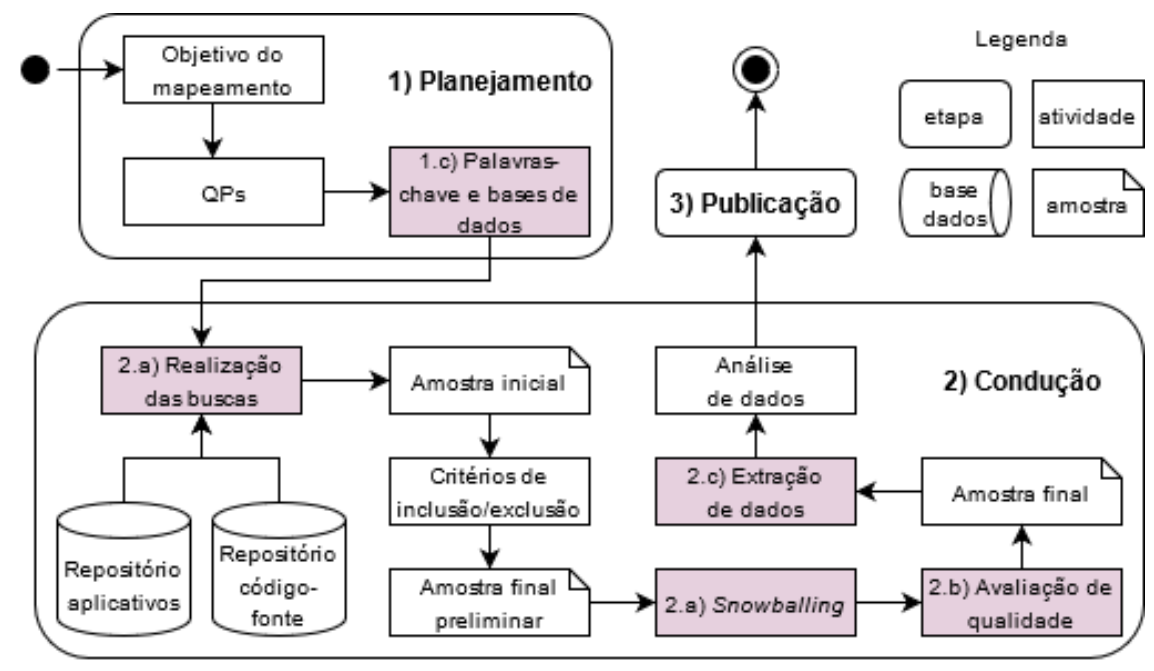

Figura 1. Visão geral do protocolo adaptado

- 1.c) definição da estratégia de buscas: os repositórios de aplicativos e de códigofonte usados para as buscas são escolhidos nesta atividade. Para o perfil de profissional da saúde sugere-se o uso do Google Play Store ${ }^{3}$, repositório oficial para Android, sendo este o sistema operacional para dispositivos móveis mais utilizado atualmente [Garg and Baliyan 2021]. Para o perfil de desenvolvedor de software sugere-se o uso do Github $^{4}$, o qual se trata do repositório para gerência, hospedagem e versionamento de código-fonte mais utilizado atualmente [Ye et al. 2021].

- 2.a) definição da estratégia de seleção: os repositórios sugeridos geralmente não permitem o uso de expressões de busca, logo deve-se realizar uma busca individual para cada palavra-chave definida para a obtenção da amostra inicial de aplicativos. Esta estratégia é utilizada em [Souza and Silva 2016, Formagini et al. 2017, Marcelo et al. 2020, Morais et al. 2020, Querino et al. 2020, Trecca et al. 2021]. As especificações técnicas do dispositivo (desktop ou móvel) usado para as buscas devem ser catalogadas, pois variações podem influenciar a análise dos aplicativos.

\footnotetext{
${ }^{3}$ Disponível em https://play.google.com

${ }^{4}$ Disponível em https://github. com
} 
Para a seleção da amostra final preliminar de aplicativos, a utilização dos CIs e CEs é proposta em dois filtros de seleção: $01^{\circ}$ filtro com a análise das informações do aplicativo contidas no repositório e o $2^{\circ}$ filtro com a análise do código-fonte ou do aplicativo em uso, após sua instalação no dispositivo. Este processo de seleção é utilizado em [Querino et al. 2020]. Opcionalmente, o snowballing pode considerar os aplicativos indicados como relacionados aos que compõem a amostra final preliminar em repositórios que disponibilizam essa função.

- 2.b) avaliação de qualidade: para a definição dos itens de qualidade no contexto da saúde sugere-se a utilização de guias de avaliação de qualidade [Kiatake et al. 2020, Lima et al. 2020] ou frameworks com parâmetros de avaliação de aplicativos de saúde [Henson et al. 2019, Stoyanov et al. 2015]. Aplicativos que não atendam algum item definido são excluídos da amostra.

- 2.c) extração de dados: as informações dos aplicativos da amostra final são catalogadas com o auxílio de um formulário de extração adaptado com os dados do(s) repositório(s) de busca escolhido(s). A extração de dados pode considerar aplicativos em tempo de desenvolvimento ou execução. Ainda, pode ser realizada diretamente do aplicativo, do código-fonte, da documentação ou de outros artefatos dos repositórios como revisões e mensagens de controle de versão.

Ao término das adaptações foram elaborados guias ${ }^{5}$ de aplicação que apresentam opções de repositórios para as buscas e o protocolo proposto em tópicos, com instruções para sua utilização e modelos de tabelas para auxiliar a catalogação dos resultados do MSL e do protocolo instanciado no contexto de pesquisa de cada perfil definido.

\section{Trabalhos relacionados}

A formalização da inclusão de LC em MSLs em engenharia de software é recente. Em [Garousi et al. 2019] é elaborado um guia para busca de LC baseado em uma metodologia de MSL considerando os mecanismos de buscas regulares como as bases de dados. O protocolo inclui checklists para verificar a necessidade desse tipo de busca em uma pesquisa, para indicar o momento do término das buscas e para realizar a avaliação da qualidade da literatura encontrada. O presente trabalho também adapta um protocolo de MSL, mas considera os repositórios de aplicativos e código-fonte como as bases de dados e as soluções de software hospedadas nestes repositórios como LC.

Na literatura são encontrados trabalhos que fazem buscas de aplicativos de saúde sobre tratamento do tabagismo [Formagini et al. 2017], informações nutricionais para gestantes [Brown et al. 2020], Diabetes Mellitus [Marcelo et al. 2020], hipertensão arterial sistêmica [Marcelo et al. 2020] e ansiedade e depressão [Querino et al. 2020]. Buscas de aplicativos em repositórios são também realizadas como complemento a revisões da literatura sobre as atividades da vida diária de idosos [Souza and Silva 2016], o uso de serious games na educação de higiene bucal infantil [Morais et al. 2020] e otorrinolaringologia [Trecca et al. 2021]. Destes trabalhos, apenas em [Querino et al. 2020] é citado o uso de um protocolo de MSL como apoio para a identificação de aplicativos em repositórios, com uma adaptação parcial do protocolo sem incluir etapas de snowballing e avaliação de qualidade. O presente trabalho difere-se pois considera todas as etapas envolvidas em um MSL baseando-se em diretrizes previamente definidas e consolidadas.

\footnotetext{
${ }^{5}$ Os guias estão disponíveis em https ://zenodo.org/record/5256159\#.YSYwut 9v_IV
} 


\section{Considerações finais}

Este trabalho propôs adaptações em um protocolo de MSL buscando trazer os benefícios destes estudos em buscas de aplicativos de saúde em repositórios de aplicativos e códigofonte. As adaptações foram baseadas em uma metodologia de MSL de engenharia de software com diretrizes consolidadas comumente utilizada em pesquisas de computação e fundamentadas com abordagens de levantamento de aplicativos de saúde encontradas na literatura. Como resultado preliminar, foram elaborados dois guias para auxiliar profissionais de saúde e desenvolvedores de software no planejamento e condução de um MSL. Apesar do direcionamento apresentado para a área de saúde, ressalta-se que o protocolo proposto pode ser utilizado em outros domínios de aplicação. Como continuidade deste trabalho, será conduzido um estudo com objetivos, sujeitos e metodologia definidos visando avaliar a viabilidade e realizar melhorias no protocolo e nos guias de aplicação.

\section{Agradecimentos}

Ao PPGInf-CAPES/MEC e PET/MEC, pelo apoio financeiro.

\section{Referências}

Basili, V. R. and Rombach, H. D. (1988). The tame project: Towards improvementoriented software environments. IEEE Transactions on SE, 14(6):758-773.

Brasil, L. M. (2008). Informática em saúde. In Informática em Saúde, pages 574-574.

Brown, H. M., Bucher, T., Collins, C. E., and Rollo, M. E. (2020). A review of pregnancy apps freely available in the google play store. Health Promotion Journal of Australia.

Formagini, T. D. B., Ervilha, R. R., Machado, N. M., Andrade, B. A. B. B. d., Gomide, H. P., and Ronzani, T. M. (2017). Revisão dos aplicativos de smartphones para cessação do tabagismo disponíveis em língua portuguesa. Cadernos de Saúde Pública.

Garg, S. and Baliyan, N. (2021). Comparative analysis of android and ios from security viewpoint. Computer Science Review, 40:100372.

Garousi, V., Felderer, M., and Mäntylä, M. V. (2019). Guidelines for including grey literature and conducting multivocal literature reviews in software engineering. Information and Software Technology, 106:101-121.

Garousi, V., Felderer, M., Mäntylä, M. V., and Rainer, A. (2020). Benefitting from the grey literature in software engineering research. In Contemporary Empirical Methods in Software Engineering, pages 385-413. Springer.

Henson, P., David, G., Albright, K., and Torous, J. (2019). Deriving a practical framework for the evaluation of health apps. The Lancet Digital Health, 1(2):e52-e54.

Hexsel, R. A. (2002). Software livre: propostas de ações de governo para incentivar o uso do software livre. Relatório Técnico n. RT-DINF 004/2002, 14.

Kamei, F., Wiese, I., Lima, C., Polato, I., Nepomuceno, V., Ferreira, W., Ribeiro, M., Pena, C., Cartaxo, B., Pinto, G., et al. (2021). Grey literature in software engineering: A critical review. Information and Software Technology, page 106609.

Kamei, F., Wiese, I., Pinto, G., Ribeiro, M., and Soares, S. (2020). On the use of grey literature: A survey with the brazilian software engineering research community. In Proceedings of the 34th SBES, pages 183-192. 
Kiatake, L. G. G., Junior, L. A. V., da Silva, M. L., and Sanzovo, O. A. C. (2020). Manual Para Certificação de S-res. v. 5.0. Sociedade Brasileira de Inf. em Saúde.

Kitchenham, B. and Charters, S. (2007). Guidelines for performing systematic literature reviews in software engineering.

Laurent, A. M. S. (2004). Understanding open source and free software licensing: guide to navigating licensing issues in existing \& new software. O'Reilly Media, Inc.

Lima, L. F., Huve, C. A. G., and Peres, L. M. (2020). Software product quality evaluation guide for electronic health record systems. In Proceedings of the 34th Brazilian Symposium on Software Engineering, pages 108-113.

Marcelo, C. A. d. S., Coutinho, M. A. P., Lara, C. R., Paraizo, C. M. S., and Fava, S. M. C. L. (2020). Aplicativos móveis sobre diabetes mellitus - revisão narrativa. Journal of Health Informatics, 12(2).

Morais, E. R., Vergara, C. M. A. C., Brito, F. O. d., and Sampaio, H. A. d. C. (2020). Serious games para educação em higiene bucal infantil: uma revisão integrativa e a busca de aplicativos. Ciência \& Saúde Coletiva, 25:3299-3310.

Pappas, C. and Williams, I. (2011). Grey literature: its emerging importance. Journal of Hospital Librarianship, 11(3):228-234.

Petersen, K., Vakkalanka, S., and Kuzniarz, L. (2015). Guidelines for conducting systematic mapping studies in SE: An update. Information and Software Technology.

Program, G. C. (1999). Fourth international conference on grey literature: New frontiers in grey literature. greynet.

Querino, J. d. J., Andrade, N. N., Santos, G. B. d., and Santos, L. C. (2020). Levantamento e avaliação de aplicativos sobre ansiedade e depressão em língua portuguesa.

Reynolds, C. J. and Wyatt, J. C. (2011). Open source, open standards, and health care information systems. Journal of Medical Internet Research, 13(1):e24.

Souza, C. M. d. and Silva, A. N. (2016). Aplicativos para smartphones e sua colaboração na capacidade funcional de idosos.

Stoyanov, S. R., Hides, L., Kavanagh, D. J., Zelenko, O., Tjondronegoro, D., and Mani, M. (2015). Mobile app rating scale: a new tool for assessing the quality of health mobile apps. JMIR mHealth and uHealth, 3(1):e27.

Trecca, E. M. C., Lonigro, A., Gelardi, M., Kim, B., and Cassano, M. (2021). Mobile applications in otolaryngology: A systematic review of the literature, apple app store and the google play store. Annals of Otology, Rhinology \& Laryngology, 130(1):78-91.

Wohlin, C. (2014). Guidelines for snowballing in systematic literature studies and a replication in software engineering. In Proceedings of the 18th international conference on evaluation and assessment in software engineering, pages 1-10.

Yasin, A., Fatima, R., Wen, L., Afzal, W., Azhar, M., and Torkar, R. (2020). On using grey literature and google scholar in systematic literature reviews in software engineering. IEEE Access, 8:36226-36243.

Ye, X., Zheng, Y., Aljedaani, W., and Mkaouer, M. W. (2021). Recommending pull request reviewers based on code changes. Soft Computing, 25(7):5619-5632. 